\title{
URINARY CHOLESTEROL IN FOLLOW-UP OF PATIENTS WITH UROLOGIC CARCINOMAS OR BENIGN PROSTATIC HYPERPLASIA
}

\author{
D. JÜNGST, M.D. \\ R. TAUBER, M.D. \\ E. LAZIK, M.D. \\ H. J. KARL, M.D.
}

From the Divisions of Medicine II and Urology,

Klinikum Grosshadern, University of Munich, F.R. Germany

\begin{abstract}
Urinary cholesterol was determined in follow-up in 2 patients with prostatic adenoma, 3 patients with prostatic carcinoma, 3 patients with carcinoma of bladder, and 4 patients with carcinoma of kidney. All patients revealed marked urinary cholesterol hyperexcretion prior to surgery. Except forl patient with prostatic carcinoma, excretion of urinary cholesterol normalized at least six weeks after resection. In 1 remarkable case a localized recurrence of a transurethrally resected bladder tumor was associated with a marked increase of urinary cholesterol excretion six weeks prior to cystoscopy. The results point to a possible future value of this method in monitoring patients with bladder tumors, although additional studies are necessary.
\end{abstract}

Hyperexcretion of urinary cholesterol in carcinomas of prostate, kidney, and bladder has been described recently even in early clinical stages of these diseases. ${ }^{1-5}$ However, the diagnostic value of this parameter seemed to be diminished by the fact that elevated concentrations of urinary cholesterol have been observed in other, nonmalignant diseases of the kidney and urinary tract obstruction, especially prostatic adenoma with residual urine ${ }^{3,5}$ Nevertheless, serial determinations of urinary cholesterol could be useful in the monitoring of the clinical course which might be of practical value especially in patients with carcinoma of the bladder.

\section{Material and Methods}

Total urinary cholesterol was analyzed in 2-ml. aliquots of twenty-four-hour urine with a gas-liquid chromatographic assay using 4-androstenedione as internal standard and peak-hcight ratio technique for quantitation. This method has been described in detail previously. ${ }^{3}$
The preliminary study included a total of 12 patients, 10 males and 2 females, with an age range of from fifty-three to seventy-six years. The diagnosis of cancer or benign prostatic hyperplasia was proved histopathologically after resection. Only patients with marked urinary cholesterol hyperexcretion prior to surgery were selected for the study.

\section{Results}

\section{Prostatic adenoma}

Serial determinations of urinary cholesterol were performed in 2 patients with prostatic adenoma and residual urine. A marked elevation of urinary cholesterol (normal range 0.3 to $3.0 \mathrm{mg} . / 24$ hours, 2 SD) was measured in both cases prior to transurethral resection. After an interval of two to four weeks, urinary cholesterol excretion had normalized and remained in the normal range during the total follow-up time (Fig. 1A). These findings were associated with normalized urinary flow. 

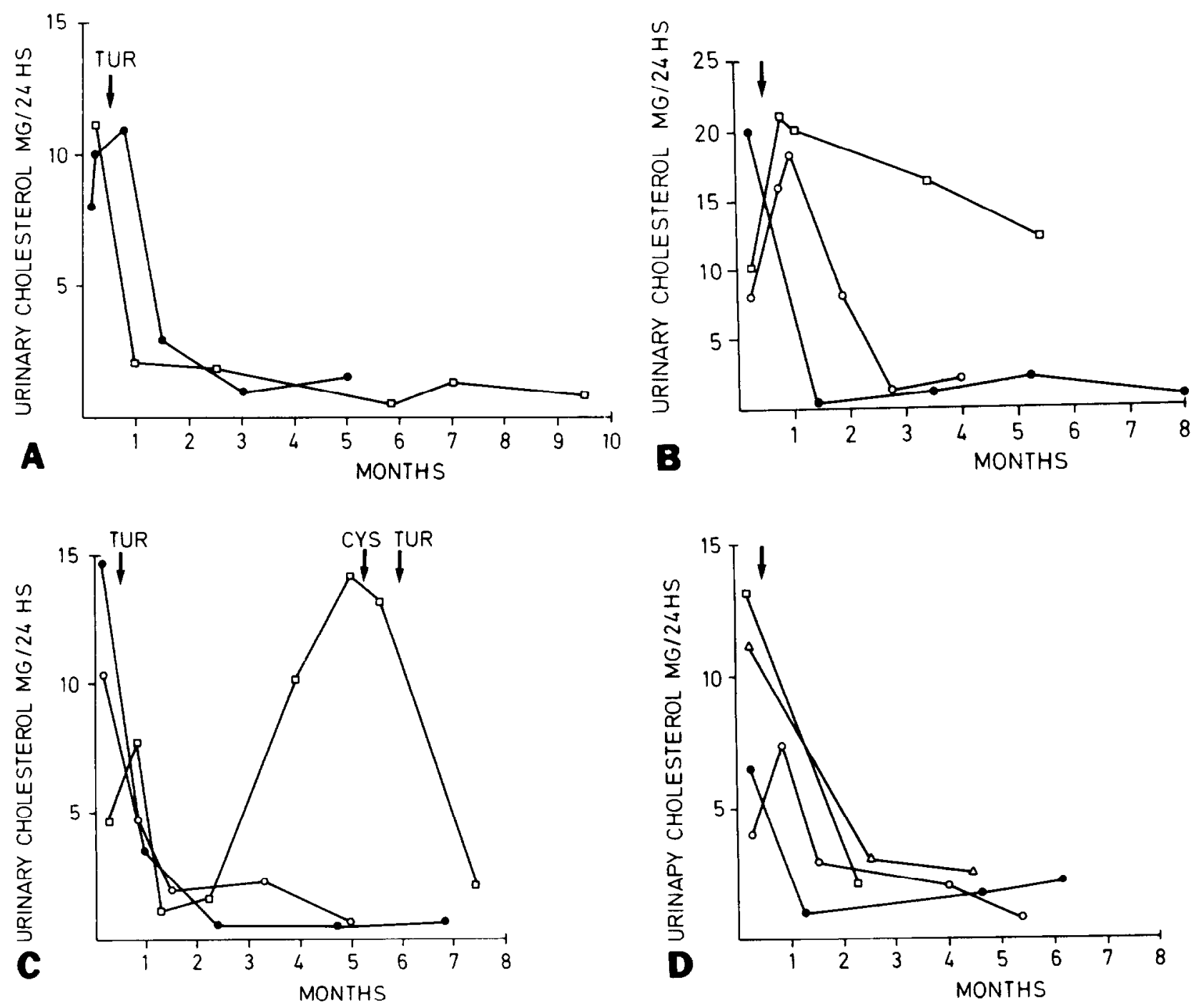

FIGURE 1. Urinary cholesterol in follow-up of (A) 2 patients with prostatic adenoma and residual urine prior to transurethral resection; (B) 3 patients with prostatic carcinoma; (C) 3 patients with carcinoma of bladder; (D) 4 patients with carcinoma of kidney.

\section{Prostatic carcinoma}

Three patients with prostatic carcinoma could be monitored by urinary cholesterol determinations. In 2 patients increments of urinary cholesterol excretion was observed up to six weeks after resection which normalized in 1 patient and remained in the other patient, most likely due to persistent obstruction of the urinary tract. A drop of urinary cholesterol was observed in the third patient four weeks after resection which remained normal until the end of the follow-up (Fig. 1B).

\section{Carcinoma of bladder}

Urinary cholesterol was measured in 3 patients with carcinoma of the bladder. All cases showed normal urinary cholesterol excretion within four weeks after transurethral resection. In 1 patient hyperexcretion of urinary cholesterol occurred three months after surgery which was due to a localized recurrence of the bladder tumor (Fig. 1C).

\section{Carcinoma of kidney}

Four patients with carcinoma of the kidney revealed slightly or marked elevations of urinary cholesterol prior to resection which normalized in all six weeks after surgery (Fig. 1D).

\section{Comment}

Increased concentrations of urinary cholesterol are usually associated with diseases of the kidney or the urogenital system and have been 
demonstrated even in early clinical stages of urologic carcinomas. ${ }^{1-12}$ In the present preliminary study serial determinations for up to eight months were performed in 12 selected patients with hyperexcretion of urinary cholesterol prior to surgery. Except for 1 patient with prostatic carcinoma, all revealed normal excretion of urinary cholesterol at least six weeks after resection. In 5 cases increments of urinary cholesterol persisted for a short time after surgery which was most likely due to urothelial lesions. In 1 remarkable case a localized recurrence of a transurethrally resected bladder tumor was associated with a marked increase of urinary cholesterol excretion six weeks prior to cystoscopy. In 2 other patients with carcinoma of the bladder urinary cholesterol levels remained normal after transurethral resection; there was no cystoscopic evidence of tumor recurrence.

These results point to a possible future value of this method in monitoring patients with bladder tumors in combination with established diagnostic procedures especially cytology. In view of this it has to be mentioned that the diagnostic sensitivity of the urinary cholesterol method is independent of the tumor grade and only slightly influenced by the tumor stage. ${ }^{3,4}$ However, additional studies in more patients are necessary to determine if urinary cholesterol measurements have a place in the monitoring of urologic diseases especially carcinomas of the bladder.
Division of Medicine II

Klinikum Grosshadern

Marchioninistr. 15

8 München 70 , F.R. Germany

(DR. JÜNGST)

References

1. Acevedo HF, et al: Urinary cholesterol V. Its excretion in men with testicular and prostatic neoplasms, Cancer 32: 196 (1973).

2. Belis JA, and Cenedella RJ: Urinary nonesterified cholesterol excretion in adenocarcinoma of the prostate, ibid. 43: 1840 (1979).

3. Jüngst $\mathrm{D}$, et al: Urinary cholesterol excretion in men with benign prostatic hyperplasia and carcinoma of the prostate, $i$ bid. 43: $361(1979)$

4. Jüngst $\mathrm{D}$, et al: Comparative evaluation of nonesterified and total urinary cholesterol in papilloma and carcinoma of the bladder, ibid. 43: 2486 (1979).

5. Pickel A: Die Bedeutung des Urincholesterins in der Diagnostik von Tumoren der Nieren, der ableitenden Harnwege und der Prostata. Thesis, LMU-München (1979).

6. Acevedo $\mathrm{HF}$, et al: Urinary cholesterol IV Its excretion in women with neoplasms of the genital system, Obstet. Gynccol. 37: 425 (1971).

7. Acevedo $\mathrm{HF}$, et al: Urinary cholesterol VII. The significance of the excretion of nonesterified cholesterol in patients with uterine carcinomas, Cancer 36: 1459 (1975).

8. Bruger M, and Ehrlich SB: Cholesterol content of the urine in patients with cancer, Arch. Intern. Med. 72: 108 (1943).

9. Burchell MM, Earle JHO, and Maclagan NF: Urinary cholesterol in cancer: urinary cholesterol excretion in cancer patients and control subjects, Br. J. Cancer 3: 42 (1949).

10. Burchell MM, and Maclagan NF: Chemical state of urinary cholesterol and methods of estimation, ibid. 3: 52 (1949).

11. Chu TM, Shukla SK, Mittelman A, and Murphy GP: Comparative evaluation of serum acid phosphatase, urinary cholesterol and androgens in diagnosis of prostatic cancer, Urology 6: 291 (1975).

12. Sobotka H, Bloch E, and Rosenbloom AB: Urinary cholesterol in cancer II, Am. J. Cancer 38: 253 (1940). 